\title{
Thermal Memory response in magneto-thermoelastic medium having long cylindrical cavity
}

\author{
SANTANU BANERJEE \\ IIEST, Shibpur \\ Department of Mathematics \\ District: Howrah, West Bengal \\ INDIA
}

\author{
BASUDEB MUKHOPADHYAY \\ IIEST, Shibpur \\ Department of Mathematics \\ District: Howrah, West Bengal \\ INDIA
}

\author{
SOUMEN SHAW \\ IIEST, Shibpur \\ Department of Mathematics \\ District: Howrah, West Bengal \\ INDIA
}

\begin{abstract}
The present paper deals with the memory response on thermal disturbances emanating from a cylindrical cavity in an unbounded thermoelastic solid. Here we have theoretically demonstrated the memory response of thermal disturbances in the generalized magneto-thermo-elastic materials. Firstly, the characteristics of thermoelastic disturbances originated from the cavity in an unbounded elastic solid under the light of generalized magnetothermoelasticity theory with memory dependent derivatives (MDD). For numerical computation, cylindrical-polar coordinate system with radial symmetry subjected to two different types of heat sources into the cavity are considered. An integral transform method and, while in inverse transformation, an efficient and pragmatic NILT (Numerical Inverse Laplace Transform) is adopted. Finally, parameter studies are performed to evaluate the effect of the kernel function and time delay. For thermal wave the results show appreciable differences with those in the usual magneto-thermoelasticity theory.
\end{abstract}

Key-Words: Thermal memory, Memory dependent derivatives, Generalized magneto-thermoelasticity, Heaviside function, Delta function, Eigen function expansion method

Received: August 19, 2019 . Revised: December 2, 2019. Accepted: December 22, 2019. Published: January 8, 2020

\section{Introduction}

The classical theory of thermoelasticity was originated with the works of Duhamel[1]. His proposed uncoupled theory was of parabolic type and it contained no terms that involved elastic deformations. There were two primary paradoxes present in that theory which was not compatible with the physical phenomena. The first paradox was that, elastic deformations doesn't produce thermal effects and the second one was that the heat conduction equation being of hyperbolic type predicted infinite velocity of thermal waves, which were contrary to the physical observations.

Over a century later, Biot [2] came upon with a classical theory of coupled thermoelasticity, which eliminated the first paradox inherent in Duhamel's uncoupled theory. Biot's theory concluded that elastic changes affect temperature. Even after the introduction of Biot's coupled theory, the second paradox in Duhamel's uncoupled theory still remained unsolved. This was due to the fact that both the theories were of diffusion type predicting infinite speeds of propagation of thermal signals. To overcome this paradox, it was essential to obtain a wave-type heat equation instead of the conventional diffusion-type. In the late sixties of the twentieth century, Lord and Shulman [3] modified the conventional classical theory by replacing the Fourier's equation of heat conduction with their newly postulated equation of heat conduction which contained one thermal relaxation time parameter connected to the heat flux vector. Lord and Shulman were the first to propose their generalization and thus introducing the generalized theory of thermoelasticity (LS theory) for the first time. Later on, Green and Lindsay (GL theory) [4] produced their generalization by modifying not only the heat conduction equation but also the equation of motion in coupled theory introducing two relaxation time parameters. This theory is often called the temperature rate dependent thermoelasticity. The heat conduction equation associated in both LS and GL theories is of hyperbolic type. Owing to such reason the generalized theories are also labeled as theories of hyperbolic thermoelasticity. During the final decade of the last century three models (Model I, II and III) concerned to the generalized theory of thermoelasticity were developed by Green and Naghdi (GN theory) [5-8]. The three models of GN theory are in accordance to three types of constitutive equations depending upon the characteristics of material response for thermal phenomena. When the 
theory is linearized, type I converges to the Fourier's classical heat equation. Type II theory predicts finite speeds for propagation of thermal waves and involves no dissipation of thermal energy, whereas the type III theory predicts an finite speed of thermal propagation and involves a thermal damping term.

Owing to the contributions made by LordShulman, Green-Lindsay and Green-Naghdi, much interest have been aroused during the last few decades towards the development of generalized thermoelastic theories. Several applications have been attempted and made in various fields of applied sciences, mathematics and engineering viz. earthquake prediction, soil dynamics, mineral exploration, etc. In the applied science and engineering problems, mathematical modeling is an important and primary task before proceeding any further. Several researchers have attempted to introduce different types of differential operators into the basic equations of thermoelasticity. One such trend was the introduction of fractional ordered derivatives into different models thermoelasticity and analyzing the subsequent results. Caputo and Mainardi[9]-[11]found a synchronization among the theoretical and experimental results. The integer ordered differential operator is a local operator whereas that fractional ordered differential operator is non local. The non local nature of the fractional derivative establishes its somewhat memory dependent nature, which is a much more realistic fitting to the real world physical problems. Thus in some recent studies fractional ordered derivatives come into account more frequently than integer ordered derivatives in various physical problems. Recently, Sherief et al.[12] and Ezzat et al.[13] came upon with their fractional models and developed the results on fractional ordered heat conduction equation in several coupled thermoelastic problems of solid mechanics.

Mathematical modeling comprises of constructing and introducing mathematical tools whose results and behaviours correspond in some way to a concerned physical system. Over the past few decades it has now become an established fact that the next state of a physical system does not only depend upon its present state but also upon all of its historical states. In the year 2011, Wang and Li [14] introduced the concept of memory dependent derivatives. This new type of derivative proved to be an useful mathematical tool and served a missing link to many physical problems. Thus as of now, parallel to fractional ordered derivatives, memory dependent derivatives serves as an important mathematical tool in describing many real world phenomenons.
During the later half of the twentieth century, nonisothermal problems played a crucial role in the development of various theories of elasticity. This was mainly due to the application and collaboration of the elasticity problems with various fields of applied sciences and engineering. One such collaboration was due to the increasing demand of research, considering the electromagnetic effects upon thermoelastic problems. For example in geophysics an important study is to analyze the effect of interactions among Earth's electromagnetic field with its plates concerning the effective changes in temperature, stress and strain giving rise to seismic waves. Among other branches such as plasma physics, nuclear engineering,electrical power engineering, rocket sciences, etc; the effect of electromagnetism upon thermoelastic models plays a vital role in the development of the respective fields.

The foundations for the study of magnetic effects upon thermoelastic problems were presented by Knopoff [15] and Chadwick [16] and developed by Kaliski and Petykiewicz [17]. In these works it was presumed that the interactions between the magnetic field and the strain field can be modeled by considering Lorentz force in the equation of motion and by a term entering Ohm's law. Eventually reporting the electric field to be produced by the velocity of a particle, moving in a magnetic field. Later Paria [18] studied the plane magneto-thermoelastic waves in an infinite isotropic body with an uniform thermal field. Paria considered the magnetic field acting transversely to the direction of propagation, whereas Wilson [19] discussed a similar problem by considering a component of magnetic field parallel to the direction of propagation. Since the last few decades of the previous century a wide attention was dedicated to the study of electromagneto thermoelastic coupled models based upon various theories of hyperbolic thermoelasticity. This is because when we have to consider short time effects, the uses of coupled parabolic thermoelasticity models produces a huge loss of accuracy. Among authors who were instrumental in studying and applying the generalized electromagneto-thermoelastic equations in the past few decades are Nayfeh and Namat-Nasser [20], Chowdhuri[21], Sherief and Ezzat[22], Ezzat et al. [23]-[26] etc.

In this paper we have considered the problem of an infinitely long elastic body having a cylindrical cavity, whose inner surface is assumed to be in a stress-free state, but subjected to only time dependent thermal disturbances. The axis of the cylindrical cavity is assumed to coincide with the $z$-axis, spread along with an axially uniform magnetic field. Such 
a problem can be considered to be an approximate replica of the situation inside the nuclear reactors, which are commonly made up of elastic materials, where heat transfer occurs through the inner surface of their cylindrical cavities.

\section{Mathematical preliminaries}

In the year 2011 Wang and $\mathrm{Li}[14]$ introduced a new class of derivatives, where he they defined the first order derivative of a function $f(x, t)$ in an integral form, in terms of normal partial derivative of $f(x, t)$ with a kernel function on a slipping interval as follows:

$$
D_{\omega}^{(1)} f(x, t)=\frac{1}{\omega} \int_{t-\omega}^{t} k(t-\xi) f_{\xi}^{\prime}(x, \xi) d \xi
$$

where $\omega$ is the time delay and $k(t-\omega)$ is the kernel function which can be chosen freely.

The above modifications to the definition of derivatives are denominated as memory dependent derivatives (MDD). Due to the form of its integrand, MDDs resonate the memory effect better than the previously used fractional ordered derivatives in several thermoelastic models, thus showing better clairvoyance in understanding the physical nature of the material.

Even though the kernel function can be chosen freely Wang and $\mathrm{Li}[14]$ indicated that to educe the memory effect better, the magnitude of MDD should be smaller than that of the common partial derivative, and for that the bounds of the kernel should be $0 \leq K(t-\xi) \leq 1$ for $\xi \in[t-\omega, t]$.

It should be noted that, following the definition of MDD, in case $K(t-\xi)=1$,

$$
\begin{gathered}
D_{\omega}^{(1)} f(x, t)=\frac{1}{\omega} \int_{t-\omega}^{t} f_{\xi}^{\prime}(x, \xi) d \xi \\
=\frac{f(x, t)-f(x, t-\omega)}{\omega}
\end{gathered}
$$

This indicates that, as $\omega \rightarrow 0$, MDD tends to common partial derivative of first order.

Recently in 2014, Yu et al.[27] introduced MDD instead of factional derivative in the generalized heat conduction law. They inserted MDD into the LordShulman (LS) generalized thermoelasticity theory in the rate of heat flux to indicate the memory reliance in the following manner:

$$
q_{i}+\tau D_{a} q_{i}=-K \theta_{, i}
$$

where $D_{a} q_{i}=D_{a}^{(1)} q_{i}=\frac{1}{a} \int_{t-a}^{t} k(t, p) q_{i}^{(1)}(p) d p$.
Nunziato [28] proposed heat conduction model with memory response at slightly different way. He assumed the response functional can memorized upto the present time of the temperature and the temperature gradient. In this model, heat conduction depends also on the present value of the temperature gradient so that Fourier's law of heat conduction is obtained as a particular case, if $k(0)$, the instantaneous conductivity, is non-zero. On the other hand, if $k(0)=0$, Nunziato's heat conduction equation agrees with that of Gurtin and Pipkin. Chen and Gurtin [29] extended the theory presented by Gurtin and Pipkin [30] to deformable media. They proposed with the constitutive assumptions that the response functional depend on the present values of the temperature and the deformation gradient and the integrated histories of the deformation gradient, temperature and the temperature gradient.

Presence of memory in the generalized thermoelasticity theories became well established since last decade of the twentieth century. Due to the presence of a time convolution integral, the Coleman-Gurtin [31] theory is referred to as the theory of heat conduction with a thermal memory. In this regard, we can mention the work of Shaw [32] a note on generalized thermoelasticity with memory dependent derivatives, in which author shown how MDDs play a significant impact in thermoelasticity theory.

The kernel $k(t, p)$ and the time delay $a(>0)$ in the definition of MDD should always be chosen in an arbitrary manner so that the material's real behavior can be understood more properly.

In common practice, the kernel function is considered in the following form:

$$
k(t, p)=\left(\frac{p-t}{a}+1\right)^{b}
$$

The following are three different forms of kernels and the respective forms of heat conduction laws:

1. Constant kernel $(b=0): q(x, t)+$ $\frac{\tau}{a}[q(x, t)-q(x, t-a)]=-K \frac{\partial \theta}{\partial x}$

2. Linear kernel $(b=1): q(x, t)+$ $\frac{\tau}{a^{2}} \int_{t-a}^{t} q(x, p) d p=-K \frac{\partial \theta}{\partial x}$

3. Parabolic kernel $(b=2): q(x, t)+$ $\frac{2 \tau}{a^{3}} \int_{t-a}^{t}(p-t+a) q(x, p) d p=-K \frac{\partial \theta}{\partial x}$. 
aic nature with $K=0$

1 for the present time $t$.

In various thermoelastic investigations, Ezzat and his co-workers [13] have proposed another form of the memory kernel as follows:

$$
k(t-p)=1-\frac{2 b}{\omega}(t-p)+\frac{a^{2}(t-p)^{2}}{\omega^{2}}
$$

where $a, b$ and $\omega$ are the parameters to be chosen.

Laplace transform of a function containing the MDD would have been a challenging task in our succeeding discussion. Thus, Laplace transform of any function $f(t)$ with first order MDD is demonstrated here,

$$
\begin{array}{r}
L\left[D_{\omega} f(t)\right]=\frac{\bar{f}(s)}{\omega}\left(1-\frac{2 b}{\omega s}+\frac{2 a^{2}}{\omega^{2} s^{2}}\right) \\
-\frac{e^{-\omega s \bar{f}(s)}}{\omega}\left(1-2 b^{2}+a^{2}+\frac{2\left(a^{2}-b\right)}{\omega s}+\frac{2 a^{2}}{\omega^{2} s^{2}}\right)
\end{array}
$$

If the kernel function in MDD is constant i.e. when $k(t, p)=1$ then,

$$
L\left[D_{\omega} f(t)\right]=\frac{1}{\omega}\left(1-e^{-\omega s}\right) \bar{f}(s)
$$

where $\bar{f}(s)$ denotes the Laplace transform of $f(t)$ and $f(t-\omega)=0$ for $t<\omega$.

\section{Basic equations}

The electrodynamics theory before Maxwell contained an inconsistency in its formulas. Maxwell corrected the inconsistency by introducing an extra term in the Ampere's law. He called the extra term the "displacement current", $\frac{\partial \mathbf{D}}{\partial t}$. Maxwell's correction to Ampere's law had an important conclusion, that a change in electric field induces a magnetic field. Henceforth all electromagnetic quantities satisfy Maxwell's equations,

$$
\begin{gathered}
\operatorname{curl} \mathbf{h}=\mathbf{J}+\frac{\partial \mathbf{D}}{\partial t} \\
\operatorname{curl} \mathbf{E}=-\frac{\partial \mathbf{B}}{\partial t} \\
\operatorname{div} \mathbf{h}=0 \\
\operatorname{div} \mathbf{E}=0
\end{gathered}
$$

$$
\begin{gathered}
\mathbf{B}=\mu_{0}\left(\mathbf{H}_{0}+\mathbf{h}\right) \\
\mathbf{D}=\varepsilon_{0} \mathbf{E}
\end{gathered}
$$

where $\mathbf{H}_{0}$ is the constant magnetic field strength, $\mathbf{h}$ is the induced magnetic field, $\mathbf{E}$ is the induced electric field, $\mathbf{J}$ is the electric current density, $\mathbf{B}$ is the magnetic induction vector, $\mathbf{D}$ is the electric induction vector, $\mu_{0}$ is the magnetic permeability and $\varepsilon_{0}$ is the electric permittivity.

In absence of inner heat sources and all other external body forces except the Lorentz force, the equation of motion has the following form:

$$
\operatorname{div} \vec{\sigma}+\mathbf{F}=\rho \frac{\partial^{2} \mathbf{u}}{\partial t^{2}}
$$

where $\vec{\sigma}$ is the stress tensor, $\rho$ is the density and $\mathbf{F}$ is an external body force also called the Lorentz's force, given by,

$$
\mathbf{F}=\mathbf{J} \times \mathbf{B}
$$

The equation of energy balance is given by,

$$
\frac{\partial}{\partial t}\left[\rho c_{e} \theta+\gamma \theta_{0} e\right]=-\operatorname{div} \mathbf{q}
$$

where $c_{e}$ is the specific heat at constant strain, $\gamma=\alpha_{t}(3 \lambda+2 \mu)$ is the material constant, $\mathbf{q}$ is the heat flux vector and $\theta_{0}$ is the reference temperature such that $\left|\frac{\theta-\theta_{0}}{\theta_{0}}\right| \ll 1$.

For a moving media, the modified Ohm's law in vector form is stated as:

$$
\mathbf{J}=\sigma_{0}\left[\mathbf{E}+\mu_{0} \frac{\partial \mathbf{u}}{\partial t} \times\left(\mathbf{H}_{0}+\mathbf{h}\right)\right]
$$

where $\sigma_{0}$ is the electric conductivity.

We may linearize the above equation by neglecting small quantities of second order, thus the Ohm's law can be reduced as,

$$
\mathbf{J}=\sigma_{0}\left[\mathbf{E}+\mu_{0} \frac{\partial \mathbf{u}}{\partial t} \times \mathbf{H}_{0}\right]
$$

The Hooke-Duhamel-Neumann law is,

$$
\sigma_{i j}=2 \mu e_{i j}+\lambda e \delta_{i j}-\gamma \theta \delta_{i j}
$$

where $\delta_{i j}$ is the Kronecker's delta and $e_{i j}$ is the strain tensor given by,

$$
e_{i j}=\frac{1}{2}\left(u_{i, j}+u_{j, i}\right)
$$


Considering the time derivative to be memory dependent we may generalize the heat conduction equation as:

$$
\mathbf{q}+\tau D_{\omega} \mathbf{q}=-k \operatorname{grad} \theta
$$

where $\omega$ is the time relaxation parameter that is to be chosen according to the requirement of the problem. get,

Using equations (18) and (19) in equation (13) we

$\mu \nabla^{2} \mathbf{u}+(\lambda+\mu) \operatorname{grad} \operatorname{div} \mathbf{u}-\gamma \operatorname{grad} \theta+\mathbf{F}=\rho \frac{\partial^{2} \mathbf{u}}{\partial t^{2}}$

Applying divergence operator on both sides of equation (21) we arrive at the following equation,

$$
(\lambda+2 \mu) \nabla^{2} e-\gamma \nabla^{2} \theta+\operatorname{div} \mathbf{F}=\rho \frac{\partial^{2} e}{\partial t^{2}}
$$

where $e$ is the cubical dilatation given by,

$$
e=\operatorname{div} \mathbf{u}
$$

Again applying divergence operator to equation (20) and using the equation of energy balance (15) we get,

$$
K \nabla^{2} \theta=\left(1+\tau D_{\omega}\right) \frac{\partial}{\partial t}\left(\rho c_{e} \theta+\gamma \theta_{0} e\right)
$$

\section{Statement of the physical problem}

Let us consider an infinitely long homogeneous, isotropic elastic body with a cylindrical cavity of radius $a_{0}$, as depicted in Figure 1.

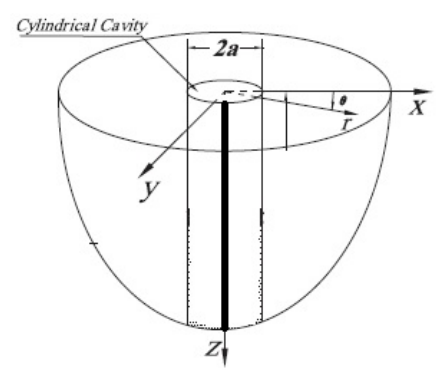

Fig. 1 Schematic diagram of the problem

The cylindrical coordinate system is taken to be $(r, \psi, z)$ with the $z$ axis being the axis of the cylindrical cavity. We consider the field variables to be $u$ and $\theta$, where $u$ is the displacement variable and $\theta$ is taken to be the temperature change above a uniform reference temperature $\theta_{0}$. The surface of the cavity is assumed to be in a stress-free state and is prone to thermal disturbances which depends only upon the time $t$. Owing to the cylindrical symmetry of the problem and if there is no $z$-dependence, all the concerned field variables will be dependent upon $r$ and $t$ only.

Hence the Laplacian operator $\nabla^{2}$ will be of the form:

$$
\nabla^{2} \equiv \frac{\partial^{2}}{\partial r^{2}}+\frac{1}{r} \frac{\partial}{\partial r}
$$

The displacement vector will be of the form,

$$
\mathbf{u}=(u, 0,0)
$$

Thus the strain tensor components are given by,

$e_{r r}=\frac{\partial u}{\partial r}, \quad e_{\psi \psi}=\frac{u}{r}, \quad e_{z z}=e_{r z}=e_{r \psi}=e_{\psi z}=0$

Hence the cubical dilatation $e$ will be of the form,

$$
e=\frac{\partial u}{\partial r}+\frac{u}{r}
$$

From equation (18) we obtain the stress tensor components as follows:

$$
\begin{array}{r}
\sigma_{r r}=2 \mu \frac{\partial u}{\partial r}+\lambda e-\gamma \theta \\
\sigma_{\psi \psi}=2 \mu \frac{u}{r}+\lambda e-\gamma \theta \\
\sigma_{z z}=\lambda e-\gamma \theta \\
\sigma_{r z}=\sigma_{z \psi}=\sigma_{\psi r}=0
\end{array}
$$

Since the constant magnetic field strength $\mathbf{H}_{0}$ acts in the direction of the $z$-axis, we consider its components to be $\left(0,0, H_{0}\right)$. Thus it can be shown that, for a conducting material the induced magnetic field $\mathbf{h}$ will have only one component in the $z$-direction and the induced electric field $\mathbf{E}$ will have only one component in the $\psi$-direction,

$$
\begin{gathered}
\mathbf{E}=\left(0, \mu_{0} H_{0} \frac{\partial u}{\partial t}, 0\right) \\
\mathbf{h}=\left(0,0,-H_{0} e\right)
\end{gathered}
$$

From equations (7), (29) and (30) we obtain the components of the electric current density as,

$$
\mathbf{J}=\left(0, H_{0}\left(\frac{\partial e}{\partial r}-\varepsilon_{0} \mu_{0} \frac{\partial^{2} u}{\partial t^{2}}\right), 0\right)
$$

From equations (11) and (30) the components of the magnetic induction vector is obtained as,

$$
\mathbf{B}=\left(0,0, \mu_{0} H_{0}(1-e)\right)
$$

Using equations (14), (31) and (32) we get the components of the Lorentz force as,

$$
\mathbf{F}=\left(\mu_{0} H_{0}^{2}\left(\frac{\partial e}{\partial r}-\varepsilon_{0} \mu_{0} \frac{\partial^{2} u}{\partial t^{2}}\right)(1-e), 0,0\right)
$$


Since $e$ is very small, we neglect the term containing the product of $e$ and hence rewrite equation (33) as,

$$
\mathbf{F}=\left(\mu_{0} H_{0}^{2}\left(\frac{\partial e}{\partial r}-\varepsilon_{0} \mu_{0} \frac{\partial^{2} u}{\partial t^{2}}\right), 0,0\right)
$$

Thus from equations (22) and (24) we obtain,

$$
\begin{aligned}
\left(1+R_{H}\right)\left(\frac{\partial^{2} e}{\partial r^{2}}+\frac{1}{r} \frac{\partial e}{\partial r}\right) & -\frac{\gamma}{\lambda+2 \mu}\left(\frac{\partial^{2} \theta}{\partial r^{2}}+\frac{1}{r} \frac{\partial \theta}{\partial r}\right) \\
& =\left(\varepsilon_{0} \mu_{0} R_{H}+\frac{1}{c_{1}^{2}}\right) \frac{\partial^{2} e}{\partial t^{2}}
\end{aligned}
$$

$$
K\left(\frac{\partial^{2} \theta}{\partial r^{2}}+\frac{1}{r} \frac{\partial \theta}{\partial r}\right)=\left(1+\tau D_{\omega}\right) \frac{\partial}{\partial t}\left(\rho C_{e} \theta+\gamma \theta_{0} e\right)
$$

where $c_{1}^{2}=\frac{\lambda+2 \mu}{\rho}$ and $R_{H}=\frac{\mu_{0} H_{0}^{2}}{\rho c_{1}^{2}}$.

We note that here $R_{H}$ is the effect of external magnetic field in the thermoelastic process proceeding in the body.

For mathematical analysis it is convenient to rewrite the governing equations in dimensionless forms. For this we shall use the following nondimensional transformations

$$
\begin{array}{r}
r^{\prime}=c_{1} \eta_{0} r, \quad u^{\prime}=c_{1} \eta_{0} u, \quad \theta^{\prime}=\frac{\gamma}{\lambda+2 \mu} \theta \\
t^{\prime}=c_{1}^{2} \eta_{0} t, \quad \tau^{\prime}=c_{1}^{2} \eta_{0} \tau, \quad \sigma_{i j}^{\prime}=\frac{1}{\mu} \sigma_{i j}
\end{array}
$$

where $\eta_{0}=\frac{\rho C_{e}}{K}$.

Thus using these transformations in equations (35), (36) and (28), and suppressing the primes for simplicity in the notations, we obtain the following equations which are in dimensionless form:

$$
\begin{gathered}
R_{M}^{2}\left(\frac{\partial^{2} e}{\partial r^{2}}+\frac{1}{r} \frac{\partial e}{\partial r}\right)-\left(\frac{\partial^{2} \theta}{\partial r^{2}}+\frac{1}{r} \frac{\partial \theta}{\partial r}\right)=R_{N}^{2} \frac{\partial^{2} e}{\partial t^{2}} \\
\left(\frac{\partial^{2} \theta}{\partial r^{2}}+\frac{1}{r} \frac{\partial \theta}{\partial r}\right)=\left(1+\tau D_{\omega}\right) \frac{\partial}{\partial t}(\theta+\varepsilon e) \\
\sigma_{r r}=\beta^{2}\left(\frac{\partial u}{\partial r}+\eta \frac{u}{r}-\theta\right) \\
\sigma_{\psi \psi}=\beta^{2}\left(\eta \frac{\partial u}{\partial r}+\frac{u}{r}-\theta\right) \\
\sigma_{z z}=\beta^{2}\left(\eta\left(\frac{\partial u}{\partial r}+\frac{u}{r}\right)-\theta\right)
\end{gathered}
$$

Here, $R_{M}^{2}=1+R_{H}, R_{N}^{2}=c_{1}^{2} \varepsilon_{0} \mu_{0} R_{H}+1$, $\varepsilon=\frac{\gamma^{2} \theta_{0}}{\rho C_{e}(\lambda+2 \mu)}, \beta^{2}=\frac{\lambda+2 \mu}{\mu}$ and $\eta=\frac{\lambda}{\lambda+2 \mu}$.

\section{Initial and boundary conditions}

The initial conditions of the problem are given by,

$$
u(r, 0)=\dot{u}(r, 0)=\theta(r, 0)=\dot{\theta}(r, 0)=0
$$

The boundary conditions can be written in the following form:

$$
\begin{gathered}
\theta\left(a_{0}, t\right)=f(t) \\
\sigma_{r r}\left(a_{0}, t\right)=0
\end{gathered}
$$

where $a_{0}$ is transformed dimensionless in accordance to equation (37) and $f(t)$ is a known function.

\section{Solution in the integral transform domain}

Performing Laplace transform upon equations (38) and (39) we obtain the following transformed governing equations for $e$ and $\theta$ :

$$
\begin{gathered}
R_{M}^{2}\left(\frac{d^{2} \bar{e}}{d r^{2}}+\frac{1}{r} \frac{d \bar{e}}{d r}\right)-\left(\frac{d^{2} \bar{\theta}}{d r^{2}}+\frac{1}{r} \frac{d \bar{\theta}}{d r}\right)=R_{N}^{2} s^{2} \bar{e} \\
\left(\frac{d^{2} \bar{\theta}}{d r^{2}}+\frac{1}{r} \frac{d \bar{\theta}}{d r}\right)=s_{1} s(\bar{\theta}+\varepsilon \bar{e})
\end{gathered}
$$

where the expression for $s_{1}$ is:

$s_{1}=1+\frac{\tau}{\omega}\left(1-e^{-s \omega}\right)$, when we consider the constant kernel form in MDD, and

$s_{1}=1+\frac{\tau}{\omega}\left(1-\frac{2 b}{\omega s}+\frac{2 a^{2}}{\omega^{2} s^{2}}\right)-$
$\frac{\tau e^{-\omega s}}{\omega}\left(1-2 b^{2}+a^{2}+\frac{2\left(a^{2}-b\right)}{\omega s}+\frac{2 a^{2}}{\omega^{2} s^{2}}\right)$, we consider the kernel form as mentioned in Ezzat et al. [?].

Considering the operator $\mathbf{L} \equiv \frac{d^{2}}{d r^{2}}+\frac{1}{r} \frac{d}{d r}$, equations (46) and (47) can be represented as,

$$
\begin{gathered}
\mathbf{L}\left[R_{M}^{2} \bar{e}-\bar{\theta}\right]=R_{N}^{2} s^{2} \bar{e} \\
\mathbf{L} \bar{\theta}=s_{1} s(\bar{\theta}+\varepsilon \bar{e})
\end{gathered}
$$

Using equation (49) upon equation (48) we obtain,

$$
\mathbf{L} \bar{e}=\frac{s}{R_{M}^{2}}\left[s_{1} \bar{\theta}+\left(R_{N}^{2} s+s_{1} \varepsilon\right) \bar{e}\right]
$$

Equations (49) and (50) can be written in the form of a vector matrix differential equation as:

$$
\mathbf{L} \tilde{v}=\tilde{A} \tilde{v}
$$


where

$$
\begin{gathered}
\tilde{A}=\left(\begin{array}{ll}
c_{11} & c_{12} \\
c_{21} & c_{22}
\end{array}\right) \\
\bar{v}=\left(\begin{array}{ll}
\bar{\theta} & \bar{e}
\end{array}\right)^{T}
\end{gathered}
$$

in which,

$$
\begin{aligned}
c_{11} & =s_{1} s, \quad c_{12}=s_{1} s \varepsilon, \\
c_{21}=\frac{s_{1} s}{R_{M}^{2}}, & c_{22}=\frac{s}{R_{M}^{2}}\left(R_{N}^{2} s+s_{1} \varepsilon\right)
\end{aligned}
$$

The characteristic equation of matrix $\tilde{A}$ can be written as:

$$
\lambda^{2}-\left(c_{11}+c_{22}\right) \lambda+\left(c_{11} c_{22}-c_{12} c_{21}\right)=0
$$

Assuming the eigenvalues of the matrix $\tilde{A}$ to be of the form: $\lambda_{1}=m_{1}^{2}$ and $\lambda_{2}=m_{2}^{2}$, the right eigen vector $\tilde{X}$ corresponding to the eigenvalue $\lambda$ of the matrix $\tilde{A}$ can be calculated as:

$$
\tilde{X}=\left(-c_{12}, c_{11}-\lambda\right)^{T}
$$

Thus one can calculate the eigen vector corresponding to the eigenvalues $\lambda_{i} \quad(i=1,2)$. We denote them as:

$$
\begin{aligned}
& \tilde{X}_{1}=\left(-c_{12}, c_{11}-m_{1}^{2}\right)^{T} \\
& \tilde{X}_{2}=\left(-c_{12}, c_{11}-m_{2}^{2}\right)^{T}
\end{aligned}
$$

In order to satisfy the regularity conditions at $r \rightarrow$ $\infty$ of the field functions, solution of equation (51) can be taken in the following form:

$$
\tilde{v}=\sum_{i=1}^{2} A_{i} \tilde{X}_{i} K_{0}\left(m_{i} r\right)
$$

where, $K_{0}($.$) being the modified Bessel function of$ second kind of $0^{\text {th }}$ order.

The transformed cubical dilatation $\bar{e}$ is given by,

$$
\bar{e}=\operatorname{div} \overline{\mathbf{u}}=\frac{1}{r} \frac{d}{d r}(r \bar{u})
$$

Integrating both sides of the above equation, we get the following relation:

$$
\bar{u}=\frac{1}{r} \int r \bar{e} d r
$$

Using equations (51) and (56) along with the relation (57) and considering the boundary conditions taken in dimensionless form, we obtain,

$$
\begin{aligned}
\bar{u}(r, s) & =\left[\frac{\xi_{2}}{m_{1} \Delta}\left(c_{11}-m_{1}^{2}\right) K_{1}\left(m_{1} r\right)\right] \bar{f}(s) \\
& -\left[\frac{\xi_{1}}{m_{2} \Delta}\left(c_{11}-m_{2}^{2}\right) K_{1}\left(m_{2} r\right)\right] \bar{f}(s) \\
\bar{\theta}(r, s) & =\frac{c_{12}}{\Delta}\left[\xi_{2} K_{0}\left(m_{1} r\right)-\xi_{1} K_{0}\left(m_{2} r\right)\right] \bar{f}(s)
\end{aligned}
$$

$$
\begin{array}{r}
\bar{\sigma}_{r r}(r, s)= \\
+\frac{\beta^{2} \xi_{1} \bar{f}(s)\left(c_{11}-m_{2}^{2}\right)(1-\eta) K_{1}\left(m_{2} r\right)}{\Delta m_{2} r} \\
-\frac{\beta^{2} \xi_{2} \bar{f}(s)\left(c_{11}+c_{12}-m_{1}^{2}\right) K_{0}\left(m_{1} r\right)}{\Delta} \\
-\frac{\beta^{2} \xi_{2} \bar{f}(s)\left(c_{11}-m_{1}^{2}\right)(1-\eta) K_{1}\left(m_{1} r\right)}{\Delta m_{1} r}
\end{array}
$$

$$
\begin{aligned}
\bar{\sigma}_{\psi \psi}(r, s)= & \frac{\beta^{2} \xi_{1} \bar{f}(s)\left(c_{12}+\eta\left(c_{11}-m_{2}^{2}\right)\right) K_{0}\left(m_{2} r\right)}{\Delta} \\
& +\frac{\beta^{2} \xi_{1} \bar{f}(s)\left(c_{11}-m_{2}^{2}\right)(\eta-1) K_{1}\left(m_{2} r\right)}{\Delta m_{2} r} \\
- & \frac{\beta^{2} \xi_{2} \bar{f}(s)\left(c_{12}+\eta\left(c_{11}-m_{1}^{2}\right)\right) K_{0}\left(m_{1} r\right)}{\Delta} \\
& -\frac{\beta^{2} \xi_{2} \bar{f}(s)\left(c_{11}-m_{1}^{2}\right)(\eta-1) K_{1}\left(m_{1} r\right)}{\Delta m_{1} r}
\end{aligned}
$$

$$
\begin{aligned}
\bar{\sigma}_{z z}(r, s) & =\frac{\beta^{2} \bar{f}(s) \xi_{1}\left(c_{12}+\eta\left(c_{11}-m_{2}^{2}\right) K_{0}\left(m_{2} r\right)\right)}{\Delta} \\
& -\frac{\beta^{2} \bar{f}(s) \xi_{2}\left(c_{12}+\eta\left(c_{11}-m_{1}^{2}\right) K_{0}\left(m_{1} r\right)\right)}{\Delta}
\end{aligned}
$$

where,

$$
\begin{gathered}
\Delta=\Omega_{2} \xi_{1}-\Omega_{1} \xi_{2} \\
\Omega_{i}=-c_{12} K_{0}\left(m_{i} a_{0}\right)
\end{gathered}
$$

$$
\begin{gathered}
\xi_{i}=\beta^{2}\left[\left(c_{11}-m_{1}^{2}\right)\left(K_{0}\left(m_{i} a_{0}\right)+\frac{1-\eta}{m_{i} a_{0}} K_{1}\left(m_{i} a_{0}\right)\right)-\Omega_{i}\right] \\
m_{i}^{2}=\frac{2 s_{1} s^{2} R_{N}^{2}}{\Upsilon+(-1)^{i+1}\left(\Upsilon^{2}-4 R_{N}^{2} R_{M}^{2} s_{1} s\right)^{\frac{1}{2}}} \\
\Upsilon=s_{1} R_{M}^{2}+R_{N}^{2} s+s_{1} \varepsilon
\end{gathered}
$$




\subsection{Applying a constant heat source at the in- ner boundary of the cylindrical wall:}

Suppose a constant step up temperature is applied in the inner surface of the cylindrical cavity, then the function $f(t)$ in equation (44) can be taken as the Heaviside function. For this the solution in Laplace transform domain will be given as:

$$
\begin{aligned}
& \bar{u}(r, s)=\frac{\xi_{2}}{\Delta s m_{1}}\left(c_{11}-m_{1}^{2}\right) K_{1}\left(m_{1} r\right) \\
& -\frac{\xi_{1}}{\Delta s m_{2}}\left(c_{11}-m_{2}^{2}\right) K_{1}\left(m_{2} r\right) \\
& \bar{\theta}(r, s)=\frac{c_{12}}{\Delta s}\left[\xi_{2} K_{0}\left(m_{1} r\right)-\xi_{1} K_{0}\left(m_{2} r\right)\right] \\
& \bar{\sigma}_{r r}(r, s)=\frac{\beta^{2} \xi_{1}\left(c_{11}+c_{12}-m_{2}^{2}\right) K_{0}\left(m_{2} r\right)}{\Delta s} \\
& +\frac{\beta^{2} \xi_{1}\left(c_{11}-m_{2}^{2}\right)(1-\eta) K_{1}\left(m_{2} r\right)}{\Delta s m_{2} r} \\
& -\frac{\beta^{2} \xi_{2}\left(c_{11}+c_{12}-m_{1}^{2}\right) K_{0}\left(m_{1} r\right)}{\Delta s} \\
& -\frac{\beta^{2} \xi_{2}\left(c_{11}-m_{1}^{2}\right)(1-\eta) K_{1}\left(m_{1} r\right)}{\Delta s m_{1} r} \\
& \bar{\sigma}_{\psi \psi}(r, s)=\frac{\beta^{2} \xi_{1}\left(c_{12}+\eta\left(c_{11}-m_{2}^{2}\right)\right) K_{0}\left(m_{2} r\right)}{\Delta s} \\
& +\frac{\beta^{2} \xi_{1}\left(c_{11}-m_{2}^{2}\right)(\eta-1) K_{1}\left(m_{2} r\right)}{\Delta s m_{2} r} \\
& -\frac{\beta^{2} \xi_{2}\left(c_{12}+\eta\left(c_{11}-m_{1}^{2}\right)\right) K_{0}\left(m_{1} r\right)}{\Delta s} \\
& -\frac{\beta^{2} \xi_{2}\left(c_{11}-m_{1}^{2}\right)(\eta-1) K_{1}\left(m_{1} r\right)}{\Delta s m_{1} r} \\
& \begin{aligned}
\bar{\sigma}_{z z}(r, s) & =\frac{\beta^{2} \xi_{1}\left(c_{12}+\eta\left(c_{11}-m_{2}^{2}\right) K_{0}\left(m_{2} r\right)\right)}{\Delta s} \\
& -\frac{\beta^{2} \xi_{2}\left(c_{12}+\eta\left(c_{11}-m_{1}^{2}\right) K_{0}\left(m_{1} r\right)\right)}{\Delta s}
\end{aligned}
\end{aligned}
$$

\subsection{Applying an instantaneous heat source at the inner boundary of the cylindrical hole:}

Sometimes inside the nuclear reactors a sudden huge rise in temperature is a very common phenomenon. Hence by considering Dirac's delta function in the boundary condition (44) we may replicate the situation of a sudden infinite impulse of temperature in the inner surface of the cylindrical cavity. For such a problem corresponding expressions for the field variables in transformed domain can be obtained just by multiplying " $s$ " to the corresponding variables as obtained in the previous subsection (6.1).

\section{Numerical results and discussion}

In order to obtain the final solutions of the physical quantities in space-time domain, we must invert the transforms given in equations (58-62). Considering the fact that the expressions for $m_{i}$ and $\Omega_{i}$ given in the preceding sections are complicated functions of $s$, the Laplace inversion for the expressions of $\bar{u}$ and $\bar{\theta}$ given in equations (58) and (59) for all values of $s$ becomes redoubtable in nature. Hence we adopt the path of deriving the numerically approximate solutions for the field equations. For inverting the Laplace transforms in the required equations we employ the algorithm for numerical inversion given by Honig and Hirdes [?].

In this method, the Laplace transform $\bar{f}(r, s)$ has the inverse $f(r, t)$, which is given by the relation

$$
f(r, t)=\frac{e^{\nu t}}{2 \pi} \int_{-\infty}^{\infty} e^{i \omega t} \bar{f}(r, s) d \omega
$$

where $s=\nu+i \omega \quad(\nu, \omega \in \mathbb{R})$

Darbin derived the approximated numerical inversion relation by expanding the function $h(r, t)=$ $e^{-\nu t} f(r, t)$ in a Fourier series in the interval $[0,2 T]$. After summing the series upto a finite number $N$, we get the approximated value for $f(r, t)$ as,

$$
\begin{array}{r}
f_{N}(r, t)=-\frac{e^{\nu t}}{2 T} \operatorname{Re}\{\bar{f}(r, \nu)\} \\
+\frac{e^{\nu t}}{T} \sum_{k=0}^{N} \operatorname{Re}\left\{\bar{f}\left(r, \nu+i \frac{k \pi}{T}\right)\right\} \cos \left(\frac{k \pi}{T} t\right) \\
-\frac{e^{\nu t}}{T} \sum_{k=0}^{N} \operatorname{Im}\left\{\bar{f}\left(r, \nu+i \frac{k \pi}{T}\right)\right\} \sin \left(\frac{k \pi}{T} t\right)
\end{array}
$$

Now, for reducing the total error we use the Korrektur method and then the $\epsilon$-algorithim for reducing the discretizational error and hence to accelerate convergence.

For the purpose of numerical evaluation in the space time domain, we choose the parameters of copper-like materials where the values of physical constants are as follows: 


$$
\begin{gathered}
\lambda=7.76 \times 10^{10} \mathrm{Nm}^{-2}, \quad \mu=3.86 \times 10^{10} \mathrm{Nm}^{-2}, \\
\rho=8954 \mathrm{kgm}^{-3}, \varepsilon=0.0168, \quad C_{T}=2, \\
a_{0}=1, \quad T_{0}=293 \mathrm{~K}, \quad C_{e}=381 \mathrm{Jkg}^{-1} \mathrm{~K}^{-1} \\
k=400 \mathrm{Wm}^{-1} \mathrm{~K}^{-1}, \quad k^{*}=300 \mathrm{Wm}^{-1} \mathrm{~K}^{-1} \mathrm{~s}^{-1}
\end{gathered}
$$

Numerical computations were carried out comparing the displacement, temperature, radial and axial stress in context MDD model with various parametric values. The comparisons are demonstrated graphically in Figures 2-13 for different values of $a$ and $b$, and also with and without magnetic effects.

Figures 2-3 represented the various displacements along the radius in comparison with various memory dependent models and the LS model, with and without magnetic effects. In Figures 2 and 3 we observed that the magnitude of displacement is maximum near the surface of the cylindrical cavity and it proportionately decreases along the radius of the elastic body. Further in Figure 3, the magnitude of displacement under magnetic effects is noted to be greater for the LS model than the MDD model $(a=0, b=0.5)$, even though they coincide to a constant value as we move away from the cavity. Figures $4-5$ showed that for all memory dependent thermoelasticity models under consideration, the temperature is maximum at the surface of the cavity and it gradually decreases as we move away radially in the near vicinity of the cavity. Figure 4 showed that the introduction of magnetic effect in the problem causes a rise in temperature near the surface of the cavity. It is observant that even though they both converge as we move away radially from the cavity, for the non-linear MDD model $(a=1, b=1)$ without magnetic effect, the fall in temperature is much more than the non-linear MDD model with magnetic effect near the vicinity of the cavity. Figure 5 depicted that the memory effect enhances an increase in temperature when compared with the LS model. Figures 6-9 exhibited that although the radial and axial stresses get maximized near the inner surface of the cavity, for different models they gradually converge to zero as we move away from the center of the cavity. The radial and axial stresses are observed to get decreased under magnetic effects. Figures 10-13 shows comparative studies between the boundary conditions of Heaviside and Dirac delta functions. The results are taken from subsections (6.1) and (6.2). In all comparisons, it is observed that the effect of delta function upon the physical changes is much more than the Heaviside boundary condition. The increase in radial and axial stress is effectively much high near the neighborhood of the cavity when we apply delta function in the boundary condition.

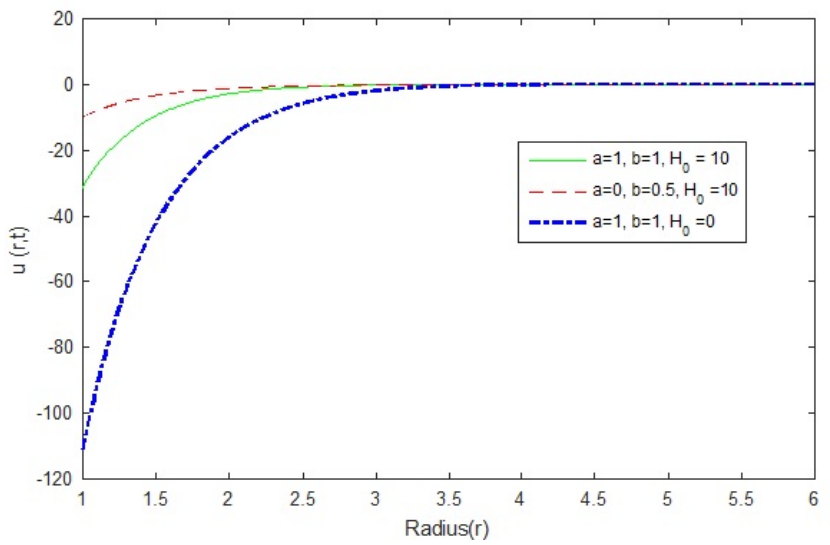

Fig. 2 Comparison of displacement $u(r, t)$ with MDD.

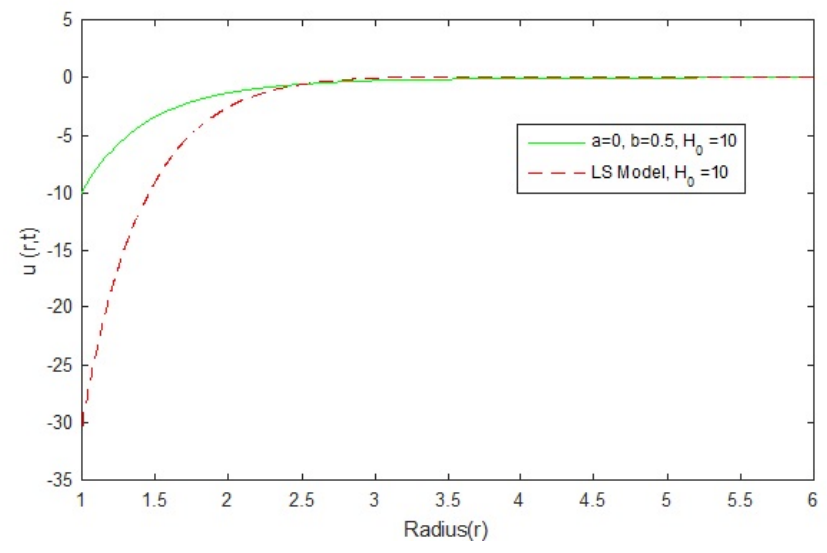

Fig. 3 Comparison of displacement $u(r, t)$ with MDD and LS model.

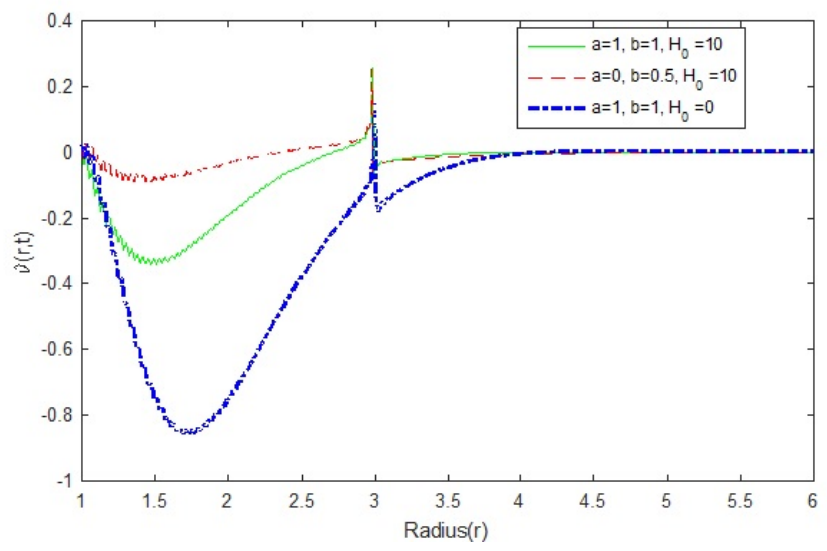

Fig. 4 Comparison of displacement $\theta(r, t)$ with MDD. 


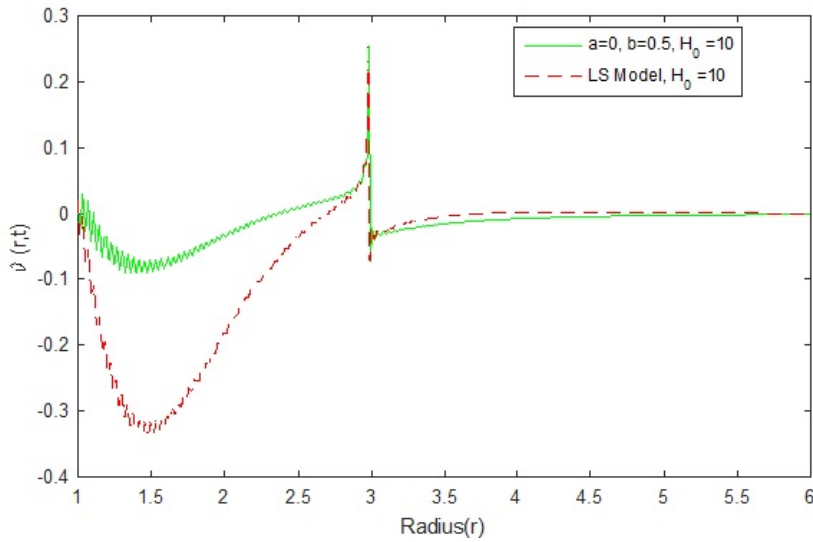

Fig. 5 Comparison of temperature $\theta(r, t)$ with MDD and LS model.

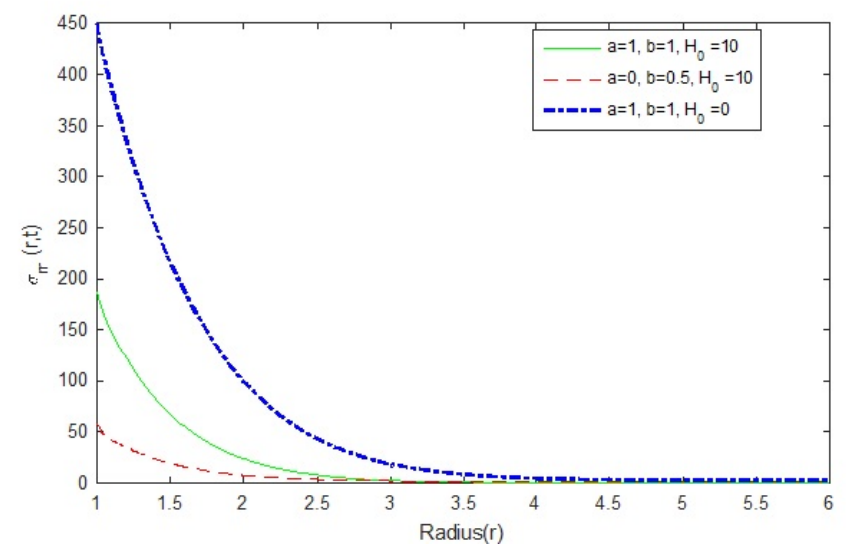

Fig. 6 Comparison of radial stresses $\sigma_{r r}(r, t)$ with MDD.

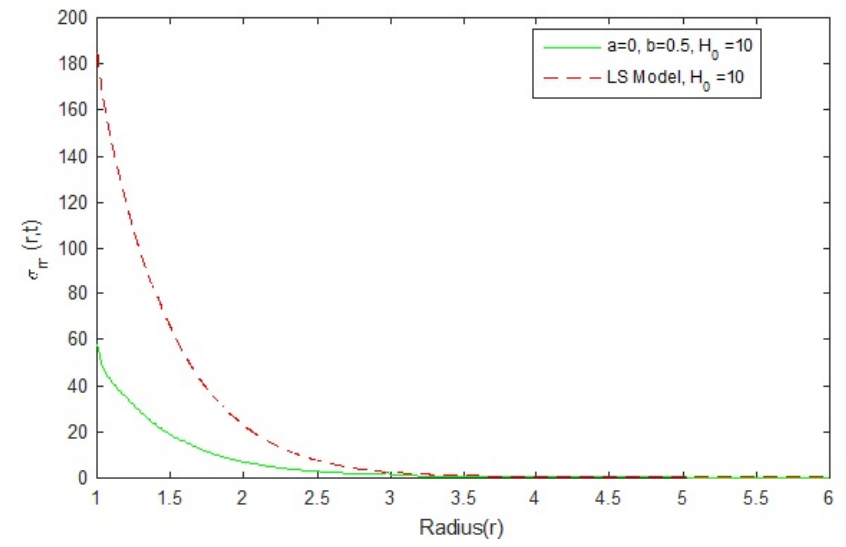

Fig. 7 Comparison of radial stresses $\sigma_{r r}(r, t)$ with MDD and LS model.

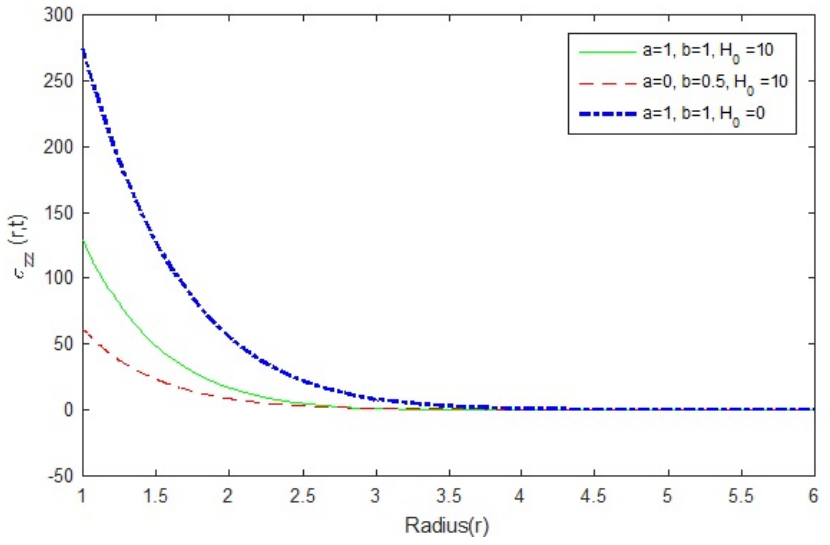

Fig. 8 Comparison of axial stresses $\sigma_{z z}(r, t)$ with MDD.

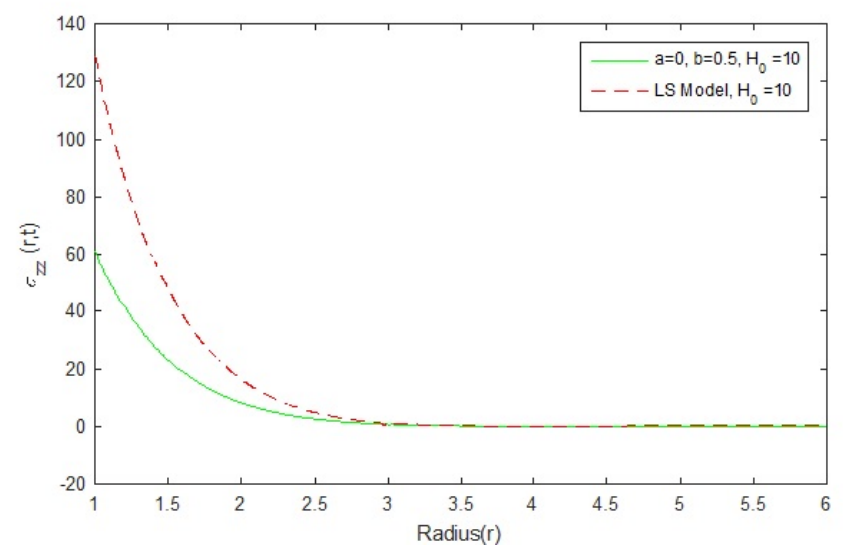

Fig. 9 Comparison of axial stresses $\sigma_{z z}(r, t)$ with MDD and LS model.

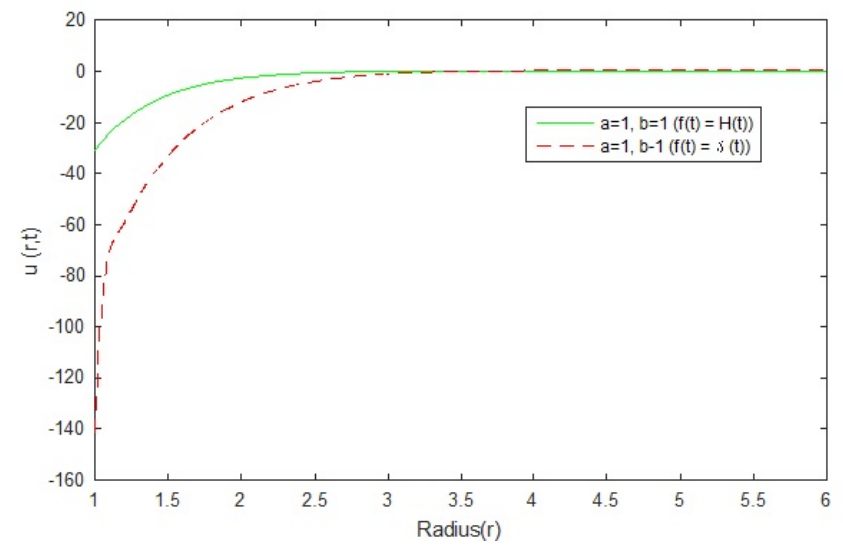

Fig. 10 Comparison of displacement $u(r, t)$ with MDD. 


\section{Conclusion}

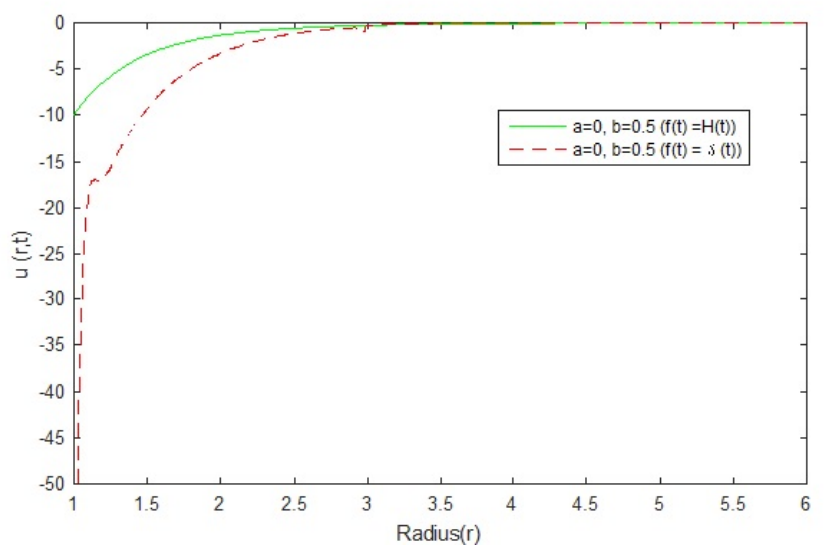

Fig. 11 Comparison of displacement $u(r, t)$ with MDD.

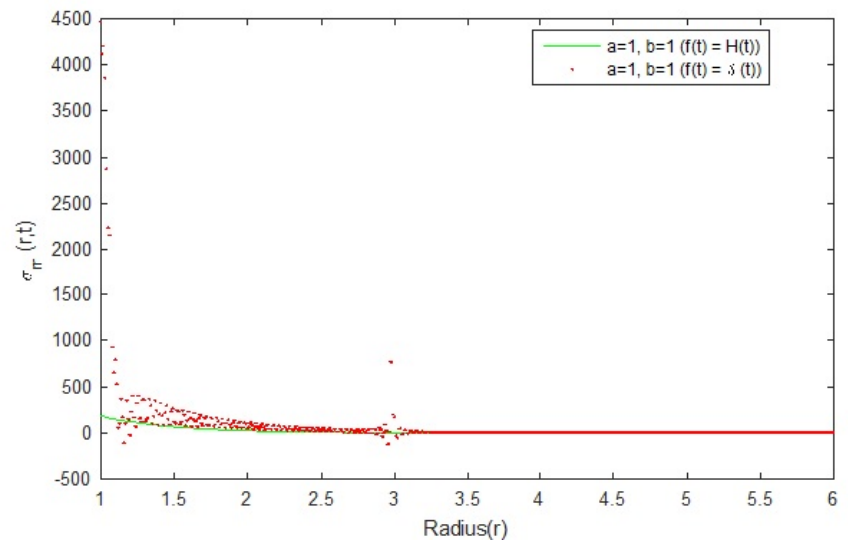

Fig. 12 Comparison of radial stresses $\sigma_{r r}(r, t)$ with MDD.

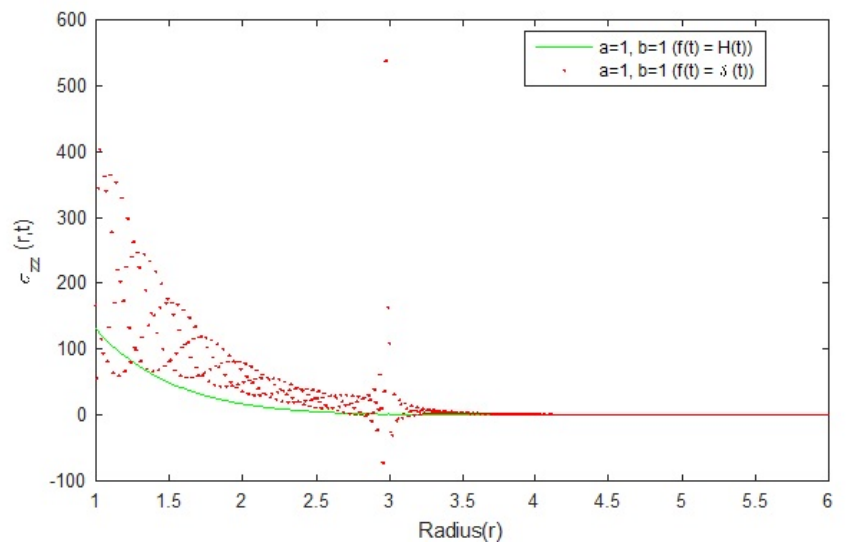

Fig. 13 Comparison of axial stresses $\sigma_{z z}(r, t)$ with MDD.
The present article provided a detailed analysis of the memory response on thermal disturbances in the context of the recently proposed generalized thermoelasticity theory with memory dependent derivatives (with and without magnetic effects). A comparative study was analyzed for the effects in physical changes in context of a constant heat source and an infinite impulsive heat source in the inner surface of the cavity. Upon selecting an appropriate kernel with the required values of the constants, one may enhance the memory effects in the theory of thermoelasticity. The influence of memory in thermoelastic models under consideration had a consequential effect over them. The graphical results suggested that the memory connected to thermal displacement was insufficient to cater the needs of non locality phenomenon in hyperbolic thermoelasticity. The graphical results revealed that the introduction of magnetic effect upon MDD models causes the drop in temperature significantly less than those without magnetic effects. The results also suggested that one can effectively reduce axial and radial stress upon the elastic body by bringing magnetic effects in the surroundings. The effect of providing an infinite impulsive temperature upon the inner boundary of the cavity revealed a significantly huge rise in radial and axial stress near the vicinity of the cavity, when compared with the condition of providing a constant step in temperature in the inner boundary of the elastic body. The analysis and results exhibited in this article may prove to be functional for researchers who are working on material science, mathematical physics, electromagnetism, thermodynamics with low temperatures as well as on the evolution of the hyperbolic thermoelasticity theory.

\section{References:}

[1] J.H. Duhamel, Second memoire, sur les phenomenes thermomechanique, J. de l'Ecole polytech. 15, 1837, pp. 1-15.

[2] M. Biot, Thermoelasticity and irreversible thermodynamics, J. Appl. Phys. 27, 1956, pp. 240253.

[3] H. Lord, Y. Shulman, A generalized dynamical theory of thermoelasticity, J. Mech. Phys. Solids. 15, 1967, pp. 299-309.

[4] A. Green, K. Lindsay, Thermoelasticity, J. Elast. 2, 1972, pp. 1-7.

[5] A.E. Green, P.M. Naghdi, A re-examination of basic postulates of thermomechanics. Proc. Roy. Soc. Ser. 432, 1991, pp. 171-194. 
[6] A.E. Green, P.M. Naghdi, On undamped heat waves in an elastic solid, J. Therm. Stress. 15, 1992, pp. 253-264.

[7] A.E. Green, P.M. Naghdi, Thermoelasticity without energy dissipation, J. Elast. 31, 1993, pp. 189-208.

[8] A.E. Green, P.M. Naghdi, A unified procedure for construction of theories of deformable media. I. Classical continuum physics, II. Generalized continua, III. Mixture of interacting continua, Proc. Roy. Soc. Lond. 448, 1995, pp. 335388.

[9] Caputo M., Mainardi F., A new dissipation model based on memory mechanism. Pure Appl Geophys. 1971;91:134-147.

[10] M. Caputo, F. Mainardi, Linear models of dissipation in elastic solids, Rivisita del Nuovo. Cimento. 1, 1971, pp. 161-198.

[11] F. Mainardi, Fractional Calculus and Waves in Linear Viscoelasticity, Imperial College Press and Singapore, World Scientific, London. 2010.

[12] H.H. Sherief, A. El-Sayed, A.A. El-Latief, Fractional order theory of thermoelasticity, Int. J. Solids Struct. 47, 2010, pp. 269-275.

[13] M.A. Ezzat, A.S. El-Karamany, M.A. Fayik, Fractional order theory in thermoelastic solid with three phase lag heat transfer, Arch. Appl. Mech. 82, 2012, pp. 557-572.

[14] J.L. Wang , H.F. Li, Surpassing the ractional derivatve: Concept of memory-dependent derivative, Comput. Math. Appl. 62, 2011, pp. 1562-1567.

[15] L. Knopoff, The interaction between elastic wave motion and a magnetic field in electrical conductors, J. Geophys. Res. 60, 1955, pp. 441456.

[16] P. Chadwick, Ninth Int. Congr. Appl Mech. 7, 1957, pp. 143.

[17] S. Kaliski, J. Petykiewicz, Equation of motion coupled with the field of temperature in a magnetic field involving mechanical and electrical relaxation for anisotropic bodies, Proc. Vibr. Probl. 4, 1959, pp. 1.

[18] G. Paria, On magneto-thermoelastic plane waves, Proc. Cambr. Phil. Soc. 56, 1962, pp. 527-531.

[19] A. Wilson, Proc. Cambr. Phil. Soc. 59, 1963, pp. 483-488.

[20] A. Nayfeh, S. Namat-Nasser, Electromagnetothermoelastic plane waves in solids relaxation, J. Appl. Mech. E. 39, 1972, pp. 108-113.

[21] S. Chowdhuri, Electromagneto-thermoelastic plane waves in rotating media with thermal relaxation, Int. J. Eng. Sci. 22, 1984, pp. 519-530.
[22] H. Sherief, M.A. Ezzat, Thermal shock problem in magneto-thermoelasticity with thermal relaxation, Int. J. Solid. Struct. 33, 1996, pp. 44494469.

[23] M. Ezzat, M. Othman, Electromagnetothermoelastic waves with two-relaxation times in a medium of perfect conductivity, Int. J. Eng. Sci. 38, 2000, pp. 107-120.

[24] M. Ezzat, M. Othman, A. El-Karamany, Electromagneto-thermoelastic plane waves with thermal relaxation time in a medium of perfect conductivity, J. Therm. Stress. 24, 2001, pp. 411432.

[25] M. Ezzat, M. Othman, A. El-Karamany, State space approach to two-dimensional electromagnetic-thermo-elastic problem with two-relaxation times, Int. J. Eng. Sci. 39, 2000, pp. 1383-1404.

[26] M. Ezzat, M. Othman, State apace approach to generalize magnetothermoelasticity with thermal relaxation in a medium of perfect conductivity, J. Therm. Stress. 25, 2002, pp. 409-429.

[27] Y-J. Yu , W. Hu, X-G. Tian, A novel generalized thermoelasticity model based on memorydependent derivative, Int. J. Eng. Sci. 81, 2014, pp. 123-134.

[28] J.W. Nunziato, On heat conduction in materials with memory, Q. Appl. Mech. 29, 1971, pp. 187204.

[29] P.J. Chen, M.E. Gurtin, On second sound in materials with memory, Z. Angew. Math. Phys. 21, 1970, pp. 232-241.

[30] M.E. Gurtin, A.C. Pipkin, A general theory of heat conduction with finite wave speeds, Arch Ration Mech Anal. 31, 1968, pp. 113-126.

[31] B.D. Coleman, M.E. Gurtin, Equipresence and constitutive equations for rigid heat conductors, Z. Angew. Math. Phys. 18, 1967, pp. 199-208.

[32] S. Shaw, A note on the generalized thermoelasticity theory with memory dependent derivatives, J. Heat Transf. 139, 2017, pp. 092005.

[33] M.A. Ezzat, A.S. El-Karamany, A.A. El-Bary, On dual-phase-lag thermoelasticity theory with memory-dependent derivative, Mech. Adv. Mat. Struc. 24, 2017, pp. 908-916.

[34] G. Honig, U. Hirdes, A method for the numerical inversion of Laplace transforms, J. Comput. Appl. Math. 10, 1984, pp. 113-132. 